DESY 06-034

hep-ph/0603265

April, 2006

\title{
Gravitino Overproduction in Inflaton Decay
}

\author{
Masahiro Kawasaki ${ }^{1}$, Fuminobu Takahashi ${ }^{1,2}$ and T. T. Yanagida ${ }^{3,4}$ \\ ${ }^{1}$ Institute for Cosmic Ray Research, University of Tokyo, \\ Chiba 277-8582, Japan \\ ${ }^{2}$ Deutsches Elektronen Synchrotron DESY, Notkestrasse 85, \\ 22607 Hamburg, Germany \\ ${ }^{3}$ Department of Physics, University of Tokyo, \\ Tokyo 113-0033, Japan \\ ${ }^{4}$ Research Center for the Early Universe, University of Tokyo, \\ Tokyo 113-0033, Japan
}

\begin{abstract}
Most of the inflation models end up with non-vanishing vacuum expectation values of the inflaton fields $\phi$ in the true vacuum, which induce, in general, nonvanishing auxiliary field $G_{\phi}$ for the inflaton potential in supergravity. We show that the presence of nonzero $G_{\phi}$ gives rise to inflaton decay into a pair of the gravitinos and are thereby severely constrained by cosmology especially if the gravitino is unstable and its mass is in a range of $O(100) \mathrm{GeV} \sim O(10) \mathrm{TeV}$. For several inflation models, we explicitly calculate the values of $G_{\phi}$ and find that most of them are excluded or on the verge of being excluded for the gravitino mass in that range. We conclude that an inflation model with vanishing $G_{\phi}$, typically realized in a chaotic inflation, is favored in a sense that it naturally avoids the potential gravitino overproduction problem.
\end{abstract}




\section{Introduction}

The recent Wilkinson Microwave Anisotropy Probe (WMAP) three year data [1] is consistent with generic predictions in inflation theories and hence strongly supports the basic idea of inflationary universe. Now we are reaching the stage to select the specific inflation model that is favored by the observation.

In most of inflation models in supergravity (SUGRA) an inflaton field $\phi$ has a nonvanishing expectation value at the potential minimum [2]. This implies that the Kähler potential for the inflaton field $\phi$ contains linear terms of the inflaton field in the true vacuum, even if the minimal Kähler potential is assumed at the beginning. That is, the inflaton Kähler potential is written at the potential minimum as $K=c \phi+c^{*} \phi^{\dagger}+\phi \phi^{\dagger}+$ ... With the linear terms the inflaton field $\phi$ generically has a nonvanishing auxiliary field $G_{\phi}$, where $G=K+\ln |W|^{2}$ (here and in what follows a subscript $i$ denotes a derivative with respect to the field $i$ ). Here, $K$ and $W$ are the Kähler potential and superpotential, respectively. Recently, Ref. 3] has pointed out that, in the context of the moduli problem [4, the nonvanishing auxiliary field enables the decay into a pair of the gravitinos to proceed with a rate much higher than previously thought [5]. We find this decay process is (more) important in the reheating process of the inflaton, and that a stringent constraint on the $G_{\phi}$ of the inflaton potential must be satisfied to avoid an overproduction of the gravitino keeping the success of the standard cosmology, especially when the gravitino is unstable and its mass is in the range of $O(100) \mathrm{GeV}-O(10) \mathrm{TeV}$. For several inflation models, we explicitly calculate the values of $G_{\phi}$ to exemplify how severe the bound is. Among the known inflation models, a class of chaotic inflation models naturally avoids the potential gravitino overproduction problem, since $G_{\phi}$ vanishes in the vacuum.

The new constraint on the inflaton potential $G_{\phi}$ depends on the gravitino mass. In this letter, we restrict our discussion to the case of the gravitino mass $O(1)$ TeV to make our point clear. We see how severe the new constraint is compared with the cosmological constraint on thermally produced gravitinos [6]. Discussion on more general cases of the gravitino mass is straightforward, which will be given in [7]. 


\section{Inflaton decay into a pair of gravitinos}

We estimate the decay rate of an inflaton field $\phi$ into a pair of gravitinos. To be concrete, we adopt the gravity-mediated SUSY breaking scenarios, in which the gravitino mass is almost constant during and after inflation. The decay process we consider is identical to that recently calculated for the modulus decay [3]. The relevant interactions are [8]

$$
\begin{aligned}
e^{-1} \mathcal{L}= & -\frac{1}{8} \epsilon^{\mu \nu \rho \sigma}\left(G_{\phi} \partial_{\rho} \phi-G_{\bar{\phi}} \partial_{\rho} \phi^{\dagger}\right) \bar{\psi}_{\mu} \gamma_{\nu} \psi_{\sigma} \\
& -\frac{1}{8} e^{G / 2}\left(G_{\phi} \phi+G_{\bar{\phi}} \phi^{\dagger}\right) \bar{\psi}_{\mu}\left[\gamma^{\mu}, \gamma^{\nu}\right] \psi_{\nu},
\end{aligned}
$$

where $\psi_{\mu}$ is the gravitino field, and we have chosen the unitary gauge in the Einstein frame with the Planck units, $M_{P}=1$. The real and imaginary components of the inflaton field have the same decay rate at the leading order $[3]$ :

$$
\Gamma_{3 / 2} \equiv \Gamma\left(\phi \rightarrow 2 \psi_{3 / 2}\right) \simeq \frac{1}{288 \pi} \frac{\left|G_{\phi}\right|^{2}}{g_{\phi \bar{\phi}}} \frac{m_{\phi}^{5}}{m_{3 / 2}^{2} M_{P}^{2}},
$$

where we have assumed that the inflaton mass is much larger than the gravitino mass: $m_{\phi} \gg m_{3 / 2}$, and $g_{i j^{*}}=K_{i j^{*}}$ is the Kähler metric. Note that $g_{\phi \bar{\phi}}=1$ for a canonically normalized inflaton field. Thus the decay rate is enhanced by the gravitino mass in the denominator, which comes from the longitudinal component of $\psi$ as emphasized in Ref. [3].

It should be noted that the above expression for the decay rate cannot be applicable for $H>m_{3 / 2}$ [9. The decay proceeds only if the Hubble parameter $H$ is smaller than the gravitino mass, since the chirality flip of the gravitino forbids the decay to proceed otherwise. Intuitively, the gravitino is effectively massless as long as $H>m_{3 / 2}$.

We should clarify another important issue: what is the longitudinal component of the gravitino (i.e. goldstino) made of ? Similar issue was discussed in the context of the non-thermal 'gravitino' production during preheating [10, and it was concluded that the inflatino, instead of the gravitino in the low energy, was actually created [1] \#1. The reason is that the 'gravitino' production occurs in a rather early stage of the reheating just after the inflation ends, during which the energy stored in the inflationary sector

\footnotetext{
${ }^{\# 1}$ It should be noted, however, that the inflatinos produced during preheating may be partially converted to the gravitinos in the low energy, since $G_{\phi}$ is generically nonzero in the true minimum as we will show later. This effect can further constrain the inflation models, but the detailed discussion is beyond the scope of this letter.
} 
significantly contributes to the total SUSY breaking. In our case, however, the situation is completely different; the decay into the gravitinos becomes effective, since we consider a cosmological epoch, $H<m_{3 / 2}$, when the SUSY breaking contribution of the inflaton is subdominant. Thus the gravitinos produced by the inflaton through the above decay process should coincide with those in the low energy. The gravitinos produced by inflaton decay are genuine, and thereby the gravitino overproduction problem is present.

\section{Cosmological constraint on $G_{\phi}$}

We restrict ourselves to the case of unstable gravitino with mass of $O(1) \mathrm{TeV}$. This mass region of the gravitino is an interesting region for gravity-mediated SUSY breaking scenarios. In this case we have already a cosmological constraint on the reheating temperature $T_{R}$ to avoid the overproduction of gravitinos through thermal scattering [6], that is \#2,

$$
T_{R}<O\left(10^{6} \sim 10^{8}\right) \mathrm{GeV}
$$

depending on the gravitino mass and the hadronic branching ratio $B_{h}$. This may be easily achieved with sufficiently small couplings relevant for the inflaton decay. We assume that this bound on $T_{R}$ is satisfied, which means the inflaton decay rate into the standard-model particles must satisfy \#3

$$
\Gamma_{S M} \simeq\left(\frac{\pi^{2} g_{*}}{90}\right)^{\frac{1}{2}} \frac{T_{R}^{2}}{M_{P}}<O\left(10^{-6} \sim 10^{-2}\right) \mathrm{GeV},
$$

where $g_{*} \sim 200$ counts the relativistic degrees of freedom. We point out that too much gravitinos may be produced by the inflaton decay via the interaction (11), even if the above inequality is satisfied.

The gravitino-to-entropy ratio is given by

$$
Y_{3 / 2} \simeq 2 \frac{\Gamma_{3 / 2}}{\Gamma_{S M}} \frac{3}{4} \frac{T_{R}}{m_{\phi}}
$$

where we have neglected the gravitino production from thermal scattering. To keep the success of big-bang nucleosynthesis $(\mathrm{BBN})$, the gravitino abundance must satisfy [6]

$$
m_{3 / 2} Y_{3 / 2}<O\left(10^{-14} \sim 10^{-11}\right) \mathrm{GeV} .
$$

\footnotetext{
\#2 The expansion rate at the decay time is much smaller than the gravitino mass $O(1)$ TeV and hence the condition $H<m_{3 / 2}$ is satisfied.

${ }^{\# 3}$ Throughout this letter we require $\Gamma_{3 / 2} \ll \Gamma_{S M}$, otherwise the standard cosmology would be upset.
} 
From (4), (5) and (6), we obtain

$$
\Gamma_{3 / 2}<O\left(10^{-23} \sim 10^{-17}\right)\left(\frac{m_{3 / 2}}{1 \mathrm{TeV}}\right)^{-1}\left(\frac{m_{\phi}}{10^{6} \mathrm{GeV}}\right) \mathrm{GeV}
$$

or equivalently,

$$
\left|G_{\phi}\right|<O\left(10^{-4} \sim 10^{-1}\right)\left(\frac{m_{3 / 2}}{1 \mathrm{TeV}}\right)^{\frac{1}{2}}\left(\frac{m_{\phi}}{10^{6} \mathrm{GeV}}\right)^{-2} .
$$

We show this constraint in Fig. 1 together with predictions of new and hybrid inflation models to be derived in the following sections. We can see that the hybrid inflation model is excluded, while the new inflation model is on the verge. It should be noted that, in deriving the constraint (8), we have substituted the upper bound on the reheating temperature (3). Therefore, the constraint on $G_{\phi}$ becomes severer for lower $T_{R}$, proportional to $T_{R}^{1 / 2}$.

\section{Single field inflation model}

In this section we estimate $G_{\phi}$ for an inflation model in which the inflaton sector consists of a single chiral superfield $\phi$ with nonzero vacuum expectation value (VEV) in the potential minimum. In the following we assume that the inflaton mass $m_{\phi}$ is much larger than the gravitino mass. Since $G_{\phi}$ corresponds to the fractional contribution to the SUSY breaking, it is estimated by minimizing the scalar potential with the hidden sector responsible for the SUSY breaking. We assume that the hidden sector contains a chiral superfield $z$ and the superpotential is written as

$$
W=W(z)+W(\phi)
$$

where $W(z)$ and $W(\phi)$ are superpotentials for $z$ and $\phi$, respectively. For simplicity we take the minimal Kähler potential,

$$
K=|z|^{2}+|\phi|^{2}
$$

Then the scalar potential is given by

$$
V=e^{G}\left(\left|G_{z}\right|^{2}+\left|G_{\phi}\right|^{2}-3\right) .
$$




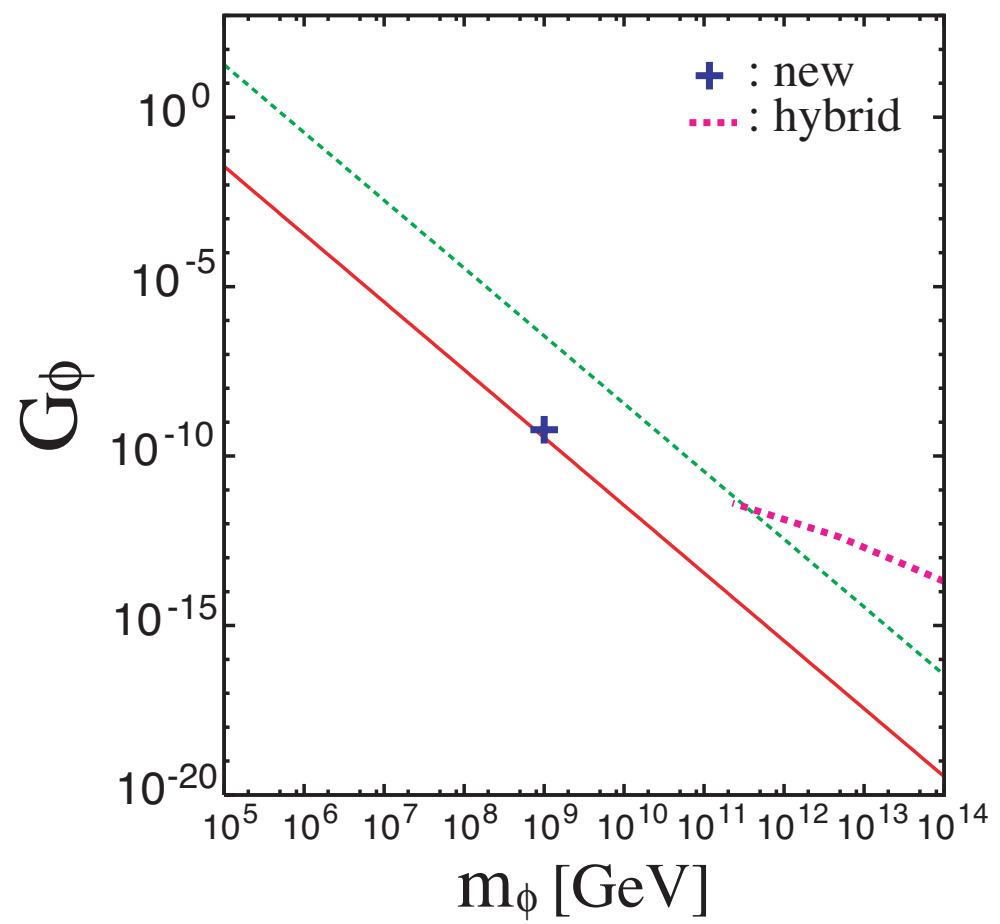

Figure 1: Upper bound on the auxiliary field of the inflaton $G_{\phi}$ as a function of the inflaton mass $m_{\phi}$. The solid and dotted lines are for the hadronic branching ratio $B_{h}=1$ and $10^{-3}$, respectively. We set $m_{3 / 2}=1 \mathrm{TeV}$. The typical values of $G_{\phi}$ and $m_{\phi}$ for the new and hybrid inflation models discussed in the text are also shown.

Since the cosmological constant should vanish in the true vacuum,

$$
\left|G_{z}\right|^{2}+\left|G_{\phi}\right|^{2}=3 .
$$

The gravitino mass is given by $m_{3 / 2}=e^{G / 2} \simeq|W|$. The potential minimum (=vacuum) is determined by a condition,

$$
V_{\phi}=e^{G}\left(G_{z \phi} G_{\bar{z}}+G_{\phi \phi} G_{\bar{\phi}}+G_{\phi}\right)=0,
$$

where we have used Eq. (12) and $G_{i \bar{j}}=\delta_{i j},(i, j=\phi, z)$. Since $z$ is responsible for the SUSY breaking, $\left|G_{z}\right| \simeq \sqrt{3}$ and $\left|W_{z}\right| \sim|W| \simeq m_{3 / 2}$. On the other hand, $\left|G_{\phi}\right| \ll 1$ for the inflaton field. Assuming $\left|G_{\phi}\right|=\left|\phi^{\dagger}+W_{\phi} / W\right| \lesssim|\phi|$, we have

$$
W_{\phi} \sim \phi W .
$$


The order estimation of Eq. (13) leads to

$$
\phi+\left(\frac{m_{\phi}}{m_{3 / 2}}-\phi^{2}\right) G_{\phi}+G_{\phi} \sim 0,
$$

where we have omitted the coefficients of order unity, and used $W_{\phi \phi} \simeq m_{\phi}$. Thus we obtain the formula for $G_{\phi}$ in the single field inflation model,

$$
\left\langle G_{\phi}\right\rangle \sim \frac{m_{3 / 2}}{m_{\phi}}\langle\phi\rangle
$$

for $m_{\phi} \gg m_{3 / 2}$. Note that this result satisfies the assumption $\left|G_{\phi}\right| \lesssim|\phi|$, so our analysis is consistent. Thus, the inflaton decay into gravitinos can set a constraint on a single field inflation model when the inflaton $\phi$ takes a non-vanishing expectation value after inflation.

For a concrete example, here we study a new inflation model [12, 13, 14]. In the new inflation model the superpotential of the inflaton sector is written as

$$
W=v^{2} \phi-\frac{\phi^{5}}{5}
$$

where $v=10^{-7} \sim 10^{-6}$ for producing the observed density fluctuations. After inflation, the inflaton $\phi$ takes the expectation value $\sim \sqrt{v}$. In this model the gravitino mass is related to $v$ as $m_{3 / 2} \sim v^{5 / 2}$, and the inflaton mass is given by $m_{\phi} \sim v^{3 / 2}$. Thus, Eq. (16) leads to $G_{\phi} \sim v^{3 / 2} \sim 6 \times 10^{-10}$ for $m_{3 / 2}=1 \mathrm{TeV}$ and $m_{\phi}=10^{9} \mathrm{GeV}$, which is close to the constraint shown in Fig. 1 ${ }^{\# 4}$.

\section{$5 \quad$ Hybrid inflation model}

In the previous section we estimate $G_{\phi}$ for single field inflation models. However, when the inflaton sector contains multiple superfields, Eq. (16) cannot be applied. Here, we consider a hybrid inflation model as a representative example.

The hybrid inflation model contains two kinds of superfields: one is $\phi$ which plays a role of inflaton and the others are waterfall fields $\Psi$ and $\tilde{\Psi}$ [16, 17, 18]. After inflation

\footnotetext{
${ }^{\# 4}$ The new inflation is also realized for $W=X\left(v^{2}-g \phi^{4}\right)$ [15] for which the constraint can be relaxed. This is because the universe after inflation is dominated by $\phi$ with suppressed $G_{\phi}$, while $G_{X}$ is not suppressed. This should be contrasted with the case of the hybrid inflation model.
} 
ends, $\phi$ as well as $\Psi$ oscillates around the potential minimum and dominates the universe until the reheating.

The total superpotential $W$ is written as

$$
W=W(z)+W(\phi, \Psi, \tilde{\Psi})
$$

where the superpotential $W(\phi, \Psi, \tilde{\Psi})$ for the inflaton sector is

$$
W(\phi, \Psi, \tilde{\Psi})=\phi\left(\mu^{2}-\lambda \tilde{\Psi} \Psi\right)
$$

Here $\lambda$ is a coupling constant and $\mu$ is the inflation energy scale. The potential minimum is located at $\langle\phi\rangle=0$ and $\langle\Psi\rangle=\langle\tilde{\Psi}\rangle=\mu / \sqrt{\lambda}$ in the SUSY limit. Including the effect of the hidden sector, however, the minimum slightly shifts as shown below. For successful inflation, $\mu$ and $\lambda$ are related as $\mu \simeq 2 \times 10^{-3} \lambda^{1 / 2}$ for $\lambda \gtrsim 10^{-3}$, and $\mu \simeq 2 \times 10^{-2} \lambda^{5 / 6}$ for $\lambda \lesssim 10^{-3}$. Moreover, in this type of hybrid inflation there exists a problem of cosmic string formation because $\Psi$ and $\tilde{\Psi}$ generally have $U(1)$ gauge charges. To avoid the problem the coupling should be small as, $\lambda \sim 10^{-4}[19]$.

Now let us estimate $G_{\phi}$ and $G_{\Psi}$. The conditions for the potential minimum lead to

$$
\begin{aligned}
& G_{z \phi} G_{\bar{z}}+G_{\phi \phi} G_{\bar{\phi}}+G_{\phi}+G_{\Psi \phi} G_{\bar{\Psi}}+G_{\tilde{\Psi} \phi} G_{\overline{\tilde{\Psi}}}=0 \\
& G_{z \Psi} G_{\bar{z}}+G_{\phi \Psi} G_{\bar{\phi}}+G_{\Psi}+G_{\Psi \Psi} G_{\bar{\Psi}}+G_{\tilde{\Psi} \Psi} G_{\overline{\tilde{\Psi}}}=0 .
\end{aligned}
$$

Here we do not use the minimization condition for $\tilde{\Psi}$, since it is equivalent to Eq. (21). In the same way as deriving Eq. (14), we assume $\left|G_{\Psi}\right| \simeq\left|G_{\tilde{\Psi}}\right| \lesssim|\Psi|$, leading to $W_{\Psi} / W \sim \Psi$. Together with Eq. (19), we obtain

$$
\langle\phi\rangle \sim \frac{m_{3 / 2}}{\lambda}
$$

where we have used $|W| \simeq m_{3 / 2}$ and $|\Psi| \simeq|\tilde{\Psi}|$. Then, with use of $G_{\Psi \phi} \sim \lambda \Psi / m_{3 / 2}-$ $\Psi W_{\phi} / W, G_{z \Psi} \sim \Psi, G_{\Psi \Psi} \sim \Psi^{2}$ and $G_{\Psi \tilde{\Psi}} \sim \lambda \phi / m_{3 / 2}-\Psi^{2}$, Eqs. (20) and (21) are written as

$$
\begin{aligned}
\frac{W_{\phi}}{W}+\frac{W_{\phi}^{2}}{W^{2}} G_{\phi}+G_{\phi}+\left(\frac{\lambda \Psi}{m_{3 / 2}}-\Psi \frac{W_{\phi}}{W}\right) G_{\Psi} & \sim 0 \\
\Psi+\left(\frac{\lambda \Psi}{m_{3 / 2}}-\Psi \frac{W_{\phi}}{W}\right) G_{\phi}+G_{\Psi}+\Psi^{2} G_{\Psi}+\left(\frac{\lambda \phi}{m_{3 / 2}}-\Psi^{2}\right) G_{\Psi} & \sim 0
\end{aligned}
$$


where we omitted coefficients of order unity. Assuming $\left|G_{\phi}\right| \lesssim|\phi|$, we obtain

$$
\begin{aligned}
&\left\langle G_{\phi}\right\rangle \sim \frac{m_{3 / 2}}{\lambda} \simeq \frac{m_{3 / 2}}{m_{\phi}}\langle\Psi\rangle \\
&\left\langle G_{\Psi}\right\rangle \simeq\left\langle G_{\tilde{\Psi}}\right\rangle \sim \frac{m_{3 / 2}^{2}}{\lambda^{2}\langle\Psi\rangle} \simeq \frac{m_{3 / 2}^{2}}{m_{\phi}^{2}}\langle\Psi\rangle .
\end{aligned}
$$

Here we have used $W_{\phi} / W \sim\langle\phi\rangle \sim m_{3 / 2} / \lambda$ and $m_{\phi}=\lambda\langle\Psi\rangle$. One can easily check that the above results satisfy the assumptions we made on $G_{\phi}$ and $G_{\Psi}$. It should be noted that $G_{\phi}$ is much larger than $G_{\Psi}$. Therefore it is $\phi$ that produces too much gravitinos.

For $m_{3 / 2}=1 \mathrm{TeV}$ and $\lambda \sim 1-10^{-4}$ we obtain $\mu \sim 2 \times 10^{-3}-10^{-5}, G_{\phi} \sim 4 \times$ $10^{-16}-4 \times 10^{-12}$ and $m_{\phi} \sim 5 \times 10^{15}-2 \times 10^{11} \mathrm{GeV}$. From Fig. 11, one can see the hybrid inflation model is excluded by the gravitino overproduction. Although the constraint on $G_{\phi}$ becomes slightly mild for $\lambda \lesssim 10^{-4}$, it is then disfavored by the WMAP result. This is because the density fluctuation becomes almost scale-invariant for $\lambda \lesssim 10^{-4}$ while the spectral index is $n_{s}=0.95 \pm 0.02$ according to the WMAP three year data 1 .

So far we have considered the standard hybrid inflation, but the final results Eqs. (25) and (26) also apply to a smooth hybrid inflation model [20, which is favored compared to the hybrid inflation model in a sense that the predicted spectral index is smaller. The constraint on this model, however, is more or less similar to the hybrid inflation, and the smooth hybrid inflation is also excluded.

\section{Chaotic inflation model}

A chaotic inflation model [21, 22] is based on a Nambu-Goldstone-like shift symmetry of the inflaton chiral multiplet $\phi$. Namely, we assume that the Kähler potential $K\left(\phi, \phi^{\dagger}\right)$ is invariant under the shift of $\phi$,

$$
\phi \rightarrow \phi+i A
$$

where $A$ is a dimensionless real parameter. Thus, the Kähler potential is a function of $\phi+\phi^{\dagger} ; K\left(\phi, \phi^{\dagger}\right)=K\left(\phi+\phi^{\dagger}\right)$. We identify its imaginary part with the inflaton field $\varphi$. Moreover, we introduce a small breaking term of the shift symmetry in the superpotential in order for the inflaton $\varphi$ to have a potential:

$$
W=m X \phi
$$


where we introduced a new chiral multiplet $X$, and $m \simeq 10^{13} \mathrm{GeV}$ determines the inflaton mass. Furthermore, we impose a discrete $Z_{2}$ symmetry which forbids the problematic linear term in Kähler potential such as $K=c\left(\phi+\phi^{\dagger}\right)+\cdots[21$, 22]. Then we take the minimal Käher potential,

$$
K=\frac{1}{2}\left(\phi+\phi^{\dagger}\right)^{2}+|X|^{2}
$$

The superpotential (28) and Kähler potential (29) lead to the scalar potential,

$$
V(\varphi, X) \simeq \frac{1}{2} m^{2} \varphi^{2}+m^{2}|X|^{2}
$$

for $|X|<1$. For $\varphi \gg 1$ and $|X|<1$, the $\varphi$ field dominates the potential and the chaotic inflation takes place [for details see Refs [21, 22]].

In this chaotic inflation model we see $G_{\phi}=0$ since $\phi=0$ and $X=0$ in the vacuum. Including the hidden sector does not modify the potential minimum due to the $Z_{2}$ symmetry. Therefore, the new gravitino problem does not exist.

\section{Conclusions}

In this letter we have shown that an inflation model with a nonzero VEV $\langle\phi\rangle \neq 0$ generically leads to the gravitino overproduction, which can jeopardize the successful standard cosmology especially when the gravitino is unstable and its mass is in the region of $O(100) \mathrm{GeV}$ and $O(10) \mathrm{TeV}$. We have explicitly calculated $G_{\phi}$, which is an important parameter to determine the gravitino abundance, for several inflation models. To be concrete we have fixed $m_{3 / 2}=1 \mathrm{TeV}$ and shown that the new inflation is on the verge of being excluded, while the hybrid inflation model is excluded. The most attractive way to get around this new gravitino problem is to have the potential minimum at the origin as a class of chaotic inflation models does. Among the known models, such a chaotic inflation model is favored in a sense that it is free from the potential gravitino overproduction problem.

Here, let us comment on other solutions. Throughout this letter we have assumed that no entropy production occurs after the reheating completes. If the huge entropy is produced at late time [23], the new gravitino problem can be greatly relaxed. Another 
even manifest solution is to assume the gravitino mass $m_{3 / 2}<\mathrm{a}$ few $\mathrm{keV} \# 5$. In this case, the produced gravitinos get into thermal equilibrium due to relatively strong interactions with the standard-model particles.

Finally, we shortly note how severe the new gravitino problem is for the gravitino mass other than $O(1) \mathrm{TeV}$. We will see that it becomes milder for a mass region $m_{3 / 2}<O(1)$ GeV suggested from gauge mediation models [25], since the cosmological constraint is weaker; it comes from the requirement that the gravitino abundance should not exceed the present dark matter abundance. For a mass region $m_{3 / 2}=O(100) \mathrm{TeV}$ suggested from anomaly mediation models [26], we will have a constraint more or less similar to the present result. The reason why we have the stringent constraint on $G_{\phi}$ for $m_{3 / 2}=$ $O(100) \mathrm{GeV}-O(10) \mathrm{TeV}$ is that the reheating temperature is so severely constrained by the $\mathrm{BBN}$ for this mass range. This means that the reheating temperature cannot be arbitrarily small even for the gravitino mass other than $O(1) \mathrm{TeV}$, since the branching ratio of the direct gravitino production increases. The extended analysis including a broad mass region for the gravitino will be given in Ref. [7.

Note added: Very recently, Dine et al [27] have pointed out that the leading term of $G_{\phi}$ is cancelled out after taking into account a mass mixing between the inflaton and the hidden-sector field $z$ and the effective $G_{\phi}^{\text {eff }}$ becomes much smaller if one takes the minimal Kähler potential. We agree with them in this point. However, as pointed out in Ref. [27], if the mass of the hidden sector field $z$ is comparable or larger than the inflaton mass, such a cancellation does not occur. In the case of the new inflation model, the inflaton mass can be comparable to the mass of $z$, and our constraint in the text is then applicable. In addition, even if the hidden-sector field mass is smaller than the inflaton mass, $G_{\phi}$ may not be suppressed if the Kähler potential is non-minimal. In fact, it is quite natural to have a non-minimal Kähler potential in the gravity-mediated SUSY breaking model. If one introduces $\delta K=\kappa / 2|\phi|^{2} z^{2}+$ h.c., the effective $G_{\phi}^{\text {eff }}$ becomes $G_{\phi}^{\text {eff }} \sim \kappa m_{3 / 2}\langle\phi\rangle / m_{\phi}$. Thus, $G_{\phi}$ in the text should be replaced by this effective $G_{\phi}^{\text {eff }}$. Notice that the hiddensector field $z$ has no charge in any symmetry and hence the above Kähler term has no

\#5 Taking account of the constraints from the CMB and the structure formation, the upper bound on the gravitino mass can be reduced to $O(10) \mathrm{eV}[24]$. 
reason to be suppressed. We find that there is still a very stringent constraint on the hybrid inflation model unless the $\kappa$ is extremely small. (For the hybrid inflation model, $G_{\phi}^{\text {eff }} \sim \kappa m_{3 / 2}\langle\Psi\rangle / m_{\phi}$ results from $\delta K=\kappa / 2|\Psi|^{2} z^{2}+$ h.c., where $\phi$ and $\Psi$ are the inflaton and the water fall field, respectively). The detailed analysis will be given elsewhere.

\section{Acknowledgments}

F.T. is grateful to Motoi Endo and Koich Hamaguchi for fruitful discussions. F.T. would like to thank the Japan Society for Promotion of Science for financial support. T.T.Y. thanks M. Ibe and Y. Shinbara for a useful discussion. The work of T.T.Y. has been supported in part by a Humboldt Research Award.

\section{References}

[1] D. N. Spergel et al., arXivastro-ph/0603449

[2] D. H. Lyth and A. Riotto, Phys. Rept. 314, 1 (1999).

[3] M. Endo, K. Hamaguchi and F. Takahashi, arXiv hep-ph/0602061;

S. Nakamura and M. Yamaguchi, arXiv:hep-ph/0602081

[4] G. D. Coughlan, W. Fischler, E. W. Kolb, S. Raby and G. G. Ross, Phys. Lett. B 131 (1983) 59;

T. Banks, D. B. Kaplan and A. E. Nelson, Phys. Rev. D 49 (1994) 779;

B. de Carlos, J. A. Casas, F. Quevedo and E. Roulet, Phys. Lett. B 318 (1993) 447.

[5] M. Hashimoto, K. I. Izawa, M. Yamaguchi and T. Yanagida, Prog. Theor. Phys. 100, 395 (1998).

[6] M. Kawasaki, K. Kohri and T. Moroi, Phys. Lett. B 625, 7 (2005); Phys. Rev. D 71, $083502(2005)$.

[7] M. Kawasaki, F. Takahashi and T.T. Yanagida, in preparation.

[8] J. Wess and J. Bagger, Supersymmetry and Supergravity, (Princeton Unversity Press, 1992).

[9] M. Endo, in private communication. 
[10] R. Kallosh, L. Kofman, A. D. Linde and A. Van Proeyen, Phys. Rev. D 61, 103503 (2000);

G. F. Giudice, I. Tkachev and A. Riotto, JHEP 9908, 009 (1999);

G. F. Giudice, A. Riotto and I. Tkachev, JHEP 9911, 036 (1999);

R. Kallosh, L. Kofman, A. D. Linde and A. Van Proeyen, Class. Quant. Grav. 17, 4269 (2000) [Erratum-ibid. 21, 5017 (2004)].

[11] H. P. Nilles, M. Peloso and L. Sorbo, Phys. Rev. Lett. 87, 051302 (2001); JHEP 0104, 004 (2001).

[12] K. Kumekawa, T. Moroi and T. Yanagida, Prog. Theor. Phys. 92, 437 (1994).

[13] K. I. Izawa and T. Yanagida, Phys. Lett. B 393, 331 (1997).

[14] M. Ibe, K. I. Izawa, Y. Shinbara and T. T. Yanagida, arXiv:hep-ph/0602192.

[15] T. Asaka, K. Hamaguchi, M. Kawasaki and T. Yanagida, Phys. Rev. D 61, 083512 (2000);

V. N. Senoguz and Q. Shafi, Phys. Lett. B 596, 8 (2004).

[16] E. J. Copeland, A. R. Liddle, D. H. Lyth, E. D. Stewart and D. Wands, Phys. Rev. D 49, 6410 (1994).

[17] G. R. Dvali, Q. Shafi and R. K. Schaefer, Phys. Rev. Lett. 73, 1886 (1994).

[18] A. D. Linde and A. Riotto, Phys. Rev. D 56, 1841 (1997).

[19] M. Endo, M. Kawasaki and T. Moroi, Phys. Lett. B 569, 73 (2003).

[20] G. Lazarides and C. Panagiotakopoulos, Phys. Rev. D 52, 559 (1995).

[21] M. Kawasaki, M. Yamaguchi and T. Yanagida, Phys. Rev. Lett. 85, 3572 (2000).

[22] M. Kawasaki, M. Yamaguchi and T. Yanagida, Phys. Rev. D 63, 103514 (2001).

[23] D. H. Lyth and E. D. Stewart, Phys. Rev. D 53, 1784 (1996);

M. Kawasaki and F. Takahashi, Phys. Lett. B 618, 1 (2005).

[24] M. Viel, J. Lesgourgues, M. G. Haehnelt, S. Matarrese and A. Riotto, Phys. Rev. D 71, 063534 (2005).

[25] G. F. Giudice and R. Rattazzi, Phys. Rept. 322, 419 (1999). 
[26] L. Randall and R. Sundrum, Nucl. Phys. B 557, 79 (1999);

G. F. Giudice, M. A. Luty, H. Murayama and R. Rattazzi, JHEP 9812, 027 (1998);

J. A. Bagger, T. Moroi and E. Poppitz, JHEP 0004, 009 (2000).

[27] M. Dine, R. Kitano, A. Morisse and Y. Shirman, arXiv hep-ph/0604140. 